COMUNICACIÓN BREVE

\title{
Impacto clínico y epidemiológico de la infección por SARS-CoV-2 en pacientes adultos con tumores sólidos
}

\author{
Recibido: 30/10/20 Aceptado: 8/11/20
}

Fabián Herrera ${ }^{1}$, Florencia Bues ${ }^{1}$, Rocío Rojas ${ }^{1}$, Elena Temporiti' , Gonzalo Recondo H. ${ }^{2}$, Pablo Mandó 2 , Clara Salgado ${ }^{3}$, Alfredo Martínez ${ }^{4}$, Cristina Videla ${ }^{4}$ y Pablo Bonvehí'.

\section{RESUMEN}

La infección por SARS-CoV-2 en pacientes con cáncer puede ser severa y con importante mortalidad. Diseñamos un estudio prospectivo observacional que tuvo como objetivo describir el impacto clínico y epidemiológico de la infección por SARS-CoV-2 en pacientes con tumores sólidos. Se incluyeron 27 pacientes con neoplasias activas, con una mediana de edad de 72 años, y mediana de score de Charlson de 6. Las infecciones fueron de adquisición nosocomial en $18,5 \%$ de los pacientes, y el $74 \%$ tuvo infiltrados pulmonares, con una alta proporción de patrón mixto y consolidativo en imágenes. Casi la mitad de los pacientes tuvo enfermedad severa y crítica. Presentaron complicaciones e infecciones hospitalarias el $26 \%$ y $14,8 \%$ respectivamente. La mortalidad a 30 días fue de $25,9 \%$, mayormente relacionada al COVID-19. La infección por SARS-CoV-2 en nuestra población tuvo considerable impacto clínico y epidemiológico.
${ }^{1}$ Sección Infectología, Departamento de Medicina del Centro de Educación Médica e Investigaciones Clínicas (CEMIC), Buenos Aires, Argentina.

${ }^{2}$ Sección Oncología, Departamento de Medicina del Centro de Educación Médica e Investigaciones Clínicas (CEMIC), Buenos Aires, Argentina.

${ }^{3}$ Sección Clínica Médica, Departamento de Medicina del Centro de Educación Médica e Investigaciones Clínicas (CEMIC), Buenos Aires, Argentina.

${ }^{4}$ Laboratorio de Virología, Departamento de Análisis Clínicos del Centro de Educación Médica e Investigaciones Clínicas (CEMIC), Buenos Aires, Argentina.

Dirección para la correspondencia: Fabián Herrera. Escobar 2657, CABA. CP: 1419.

Email: fabian1961@gmail.com

El estudio no contó con ningún tipo de financiamiento. Los contenidos del presente trabajo no han sido publicados con anterioridad. Ninguno de los autores declara presentar conflicto de intereses en relación a esta publicación.

Palabras clave: infección, SARS-CoV-2, tumores sólidos, impacto. 


\section{Introducción}

La pandemia de SARS-CoV-2 ha traído enormes consecuencias para los sistemas de salud, y particularmente para las poblaciones más vulnerables. Dentro de ellas se encuentran los pacientes con edad avanzada, aquellos con comorbilidades, obesidad, inmunocompromiso y quienes tienen cáncer $(1,2,3,4)$. Diversos estudios realizados hasta el presente en diferentes países han mostrado que la incidencia y prevalencia de SARS-CoV-2 es mayor en individuos con cáncer comparados con la población general, al igual que la presentación severa y la mortalidad $(5,6,7,8)$. No obstante, según nuestro conocimiento no hay estudios en Argentina que describan la evolución de la infección por SARS-CoV-2 en pacientes con tumores sólidos. Por dicho motivo, diseñamos el presente estudio con el objetivo de describir las características clínicas, epidemiológicas y la evolución de individuos con tumores sólidos e infección por SARS-CoV-2.

\section{Materiales y métodos}

Se trata de un estudio prospectivo y observacional realizado en un hospital universitario de la Ciudad de Buenos Aires, Argentina, especializado en la atención de pacientes con cáncer. El mismo se llevó a cabo entre junio y octubre de 2020.

Se incluyeron pacientes con edad mayor o igual a 18 años, internados con diagnóstico de un tumor sólido activo y que presentaron infección por SARS-CoV-2 al ingreso o durante su hospitalización. Se recabaron variables demográficas, epidemiológicas, clínicas, diagnósticas y de evolución. Se realizó un seguimiento de los pacientes hasta el día 30 desde el diagnóstico de la infección para evaluar mortalidad.

El diagnóstico de la infección por SARS-CoV-2 se realizó mediante una muestra de hisopado nasofaríngeo con PCR en tiempo real, utilizando el reactivo REALSTAR® SARS-COV-2 RT-PCR KIT 1.0 altona Diagnostic.

Se definió como infección respiratoria baja a aquella que presentara infiltrado pulmonar en los estudios de imágenes y/o desaturación con requerimiento de oxígeno suplementario. La severidad de la infección se categorizó mediante las definiciones propuestas en las guías de tratamiento de COVID-19 del National Institute of Health.

Se definió como infección de adquisición nosocomial a aquella cuyo diagnóstico se realizó luego de siete días de internación, no habiendo tenido síntomas al ingreso hospitalario y con una PCR previa negativa (9).
Se utilizó estadística descriptiva para caracterizar a la población de estudio. Para las variables continuas se utilizó la mediana y el rango intercuartilos (RIC). Las variables categóricas se informaron mediante la frecuencia absoluta y porcentaje.

El estudio fue aprobado por el Comité de Ética en Investigación de la institución.

\section{Resultados}

Se incluyeron 27 pacientes cuyas características demográficas y de enfermedad se encuentran descriptas en la Tabla 1. Hubo una leve predominancia de sexo femenino, con una mediana de edad elevada. También tuvieron una alta proporción de comorbilidades, con una mediana de score de Charlson de 6 (RIC: 4-10). Respecto del tratamiento del cáncer: el $44,4 \%$ se encontraba en tratamiento paliativo, $22,2 \%$ recibía quimioterapia o radioterapia y $22,2 \%$ realizó tratamiento quirúrgico el mes previo a la infección. Asimismo, recibieron quimoterapia y radioterapia en los tres meses previos un $33,3 \%$ y $18,5 \%$ respectivamente, y $14,8 \%$ recibió inmunoterapia en los seis meses previos.

La infección por SARS-CoV-2 fue mayormente de aquisición comunitaria; no obstante, en un $18,5 \%$ de los pacientes fue de adquisición nosocomial. Solo 4 pacientes habían tenido contacto con un caso confirmado de COVID-19.

La presentación clínica se describe en la Figura 1. Los síntomas más frecuentes fueron fiebre y tos con una mediana de duración de 2 días (1-12) y 7 días (2-20), respectivamente. La mediana de días desde el inicio de síntomas (casos comunitarios sintomáticos) hasta la internación fue de 1 (rango de 0-10 días).

Respecto del laboratorio de ingreso, las medianas de valores de leucocitos fueron de $6.260 / \mathrm{mm}^{3}$ (rango: $2.930-14.180$ ); PMN, 4069/ $\mathrm{mm}^{3}$ (1025- 12620); linfocitos, 1.032/ $\mathrm{mm}^{3}$ (2532.790); hemoglobina, $13 \mathrm{gr} / \mathrm{dl}(11-16)$; hematocrito, $39 \%$ (21-48), y plaquetas, $193.000 / \mathrm{mm}^{3}$ (74.000-451.000). Las medianas de los valores de los marcadores inflamatorios fueron: LDH, $446 \mathrm{UI} / \mathrm{I}$ (267-1292); PCR, $1.64 \mathrm{mg} / \mathrm{dl}$ (0.6-38.41); eritrosedimentación, $65 \mathrm{~mm} / \mathrm{hora}$ (7-126) y ferritina, $802 \mathrm{ng} /$ $\mathrm{ml}(50-5677)$.

Se realizaron imágenes de tórax en todos los pacientes: radiografía en 8 y tomografía computada en 25 . El $74 \%$ presentó infiltrados pulmonares, 2 casos evidenciados por radiografías y 18 por tomografía computada. 
Los patrones fueron vidrio esmerilado, patrón mixto (vidrio esmerilado y consolidativo) y consolidativo en $44,5 \%, 44,5 \%$ y $11,1 \%$ respectivamente. La localización fue periférica, central y difusa en $55,6 \%, 11,1 \%$ y $33,3 \%$ respectivamente.

Tabla 1. Características basales de la infección por SARS-CoV-2 en pacientes adultos con tumores sólidos

\begin{tabular}{|c|c|}
\hline Variables & n (\%) \\
\hline Edad (mediana, p25-p75) & 72 años (57-77) \\
\hline Sexo femenino & $15(55,6)$ \\
\hline \multicolumn{2}{|l|}{ Indice de masa corporal } \\
\hline$<25$ & $6(24)$ \\
\hline $25-29.9$ & $13(52)$ \\
\hline$\geq 30$ & $6(24)$ \\
\hline
\end{tabular}

\section{Comorbilidades}

\section{$\geq 2$ \\ Comorbilidades}

$12(44,4)$

Hipertensión

Diabetes

$13(48,1)$

EPOC

Insuficiencia cardiaca

$7(25,9)$

$4(14,8)$

$3(11,1)$

Enfermedad coronaria

$3(11,1)$

Asma

$2(7,4)$

Enfermedad renal crónica

Etilismo

\section{Tabaquismo}

Actual

Previo

\section{Tipo de neoplasia}

Pulmón

$11(40,7)$

Mama

$4(14,8)$

Próstata

$2(7,4)$

Tracto urinario

$2(7,4)$

Gastrointestinal alta

$2(7,4)$

Colorrectal

$2(7,4)$

SNC

$2(7,4)$

$1(3,7)$

Feocromocitoma

Paraganglioma espinal

Estadio

II

III

$8(29,6)$

$17(63)$

IV

\section{Karnofsky Score}

Mediana (p25-p75)
Se solicitaron hemocultivos en 21 pacientes (77,7\%), de los cuales solo en uno se aisló Escherichia coli. En 11 pacientes se realizó antígeno urinario para neumococo, y en 9 panel molecular para virus respiratorios en hisopado nasofaríngeo. Ambos estudios fueron negativos en todos los casos.

Respecto de la severidad, $37,1 \%$ de los casos fueron moderados, $33,3 \%$ severos, $14,8 \%$ críticos y $14,8 \%$ leves. En los pacientes con neumonía, el score PSI fue mayor de 90 (categoría de riesgo III-IV) en el 75\%, con una mediana de 102 (RIC: 91-120).

El tratamiento recibido se describe en la Figura 2. Una alta proporción de los pacientes recibió tratamiento antibiótico, y el $92,6 \%$ recibió enoxaparina ( $88 \%$ en dosis de profilaxis y $12 \%$ en dosis de tratamiento). La administración de plasma de convalesciente se administró en 10 pacientes, acorde con los protocolos de investigación vigentes en la institución.

Cuatro pacientes $(14,8 \%)$ ingresaron a terapia intensiva, de los cuales 3 requirieron asistencia ventilatoria mecánica, 2 presentaron síndrome de distrés respiratorio agudo, y solo 1 requirió drogas vasoactivas.

El 26\% presentó complicaciones durante la internación. Estas fueron: insuficiencia renal en $11,1 \%$, insuficiencia cardíaca descompensada en $11,1 \%$, arritmias y TEP en $3,7 \%$ en cada una. El 14,8\% presentó infección intrahospitalaria: infección urinaria asociada a sonda vesical en 7,4\%, neumonía asociada a ventilación mecánica y bacteriemia primaria en $3,7 \%$ cada una.

La mediana de duración de la internación en los casos comunitarios fue de 11 días (RIC: 7-23, rango de 3-30). Fallecieron 7 pacientes $(25,9 \%)$, de los cuales 5 fueron relacionadas y 2 no relacionadas al COVID-19.

Finalmente, se realizó PCR de control al día 21 en 12 pacientes, resultando negativas solo en el $66,6 \%$ de los casos.

\section{Discusión}

Este es un estudio descriptivo sobre las características epidemiológicas, clínicas y de evolución de pacientes con tumores sólidos e infección por SARS-CoV-2 atendidos en un hospital universitario de la ciudad de Buenos Aires.

Nuestra cohorte está compuesta por pacientes con una mediana de edad elevada, alta proporción de comorbilidades y de neoplasias en estadio avanzado, siendo el cáncer de 


\section{Figura 1. Presentación clínica de la infección por SARS-CoV-2}

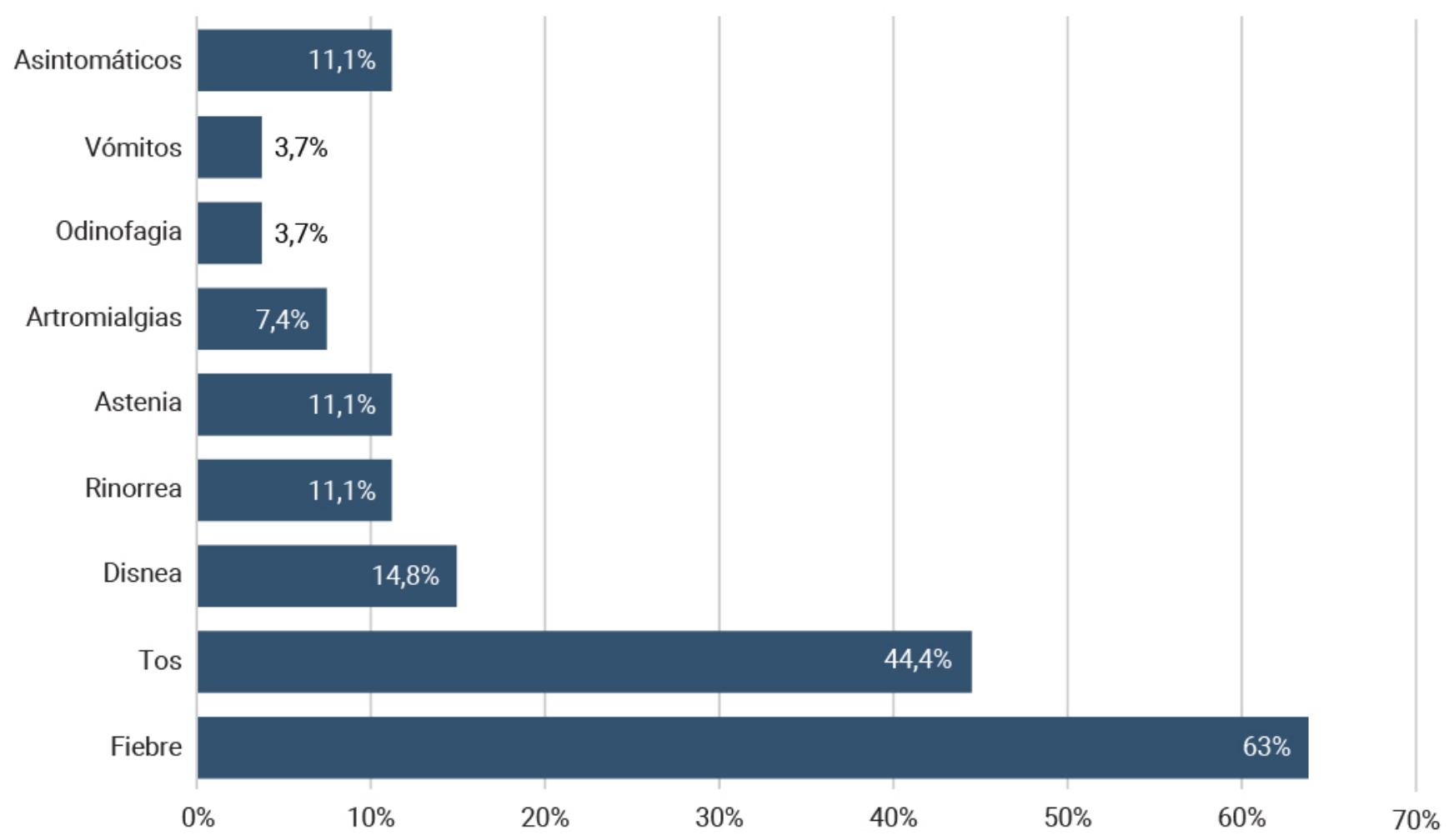

\section{Figura 2. Tratamientos recibidos para la infección por SARS-CoV-2}

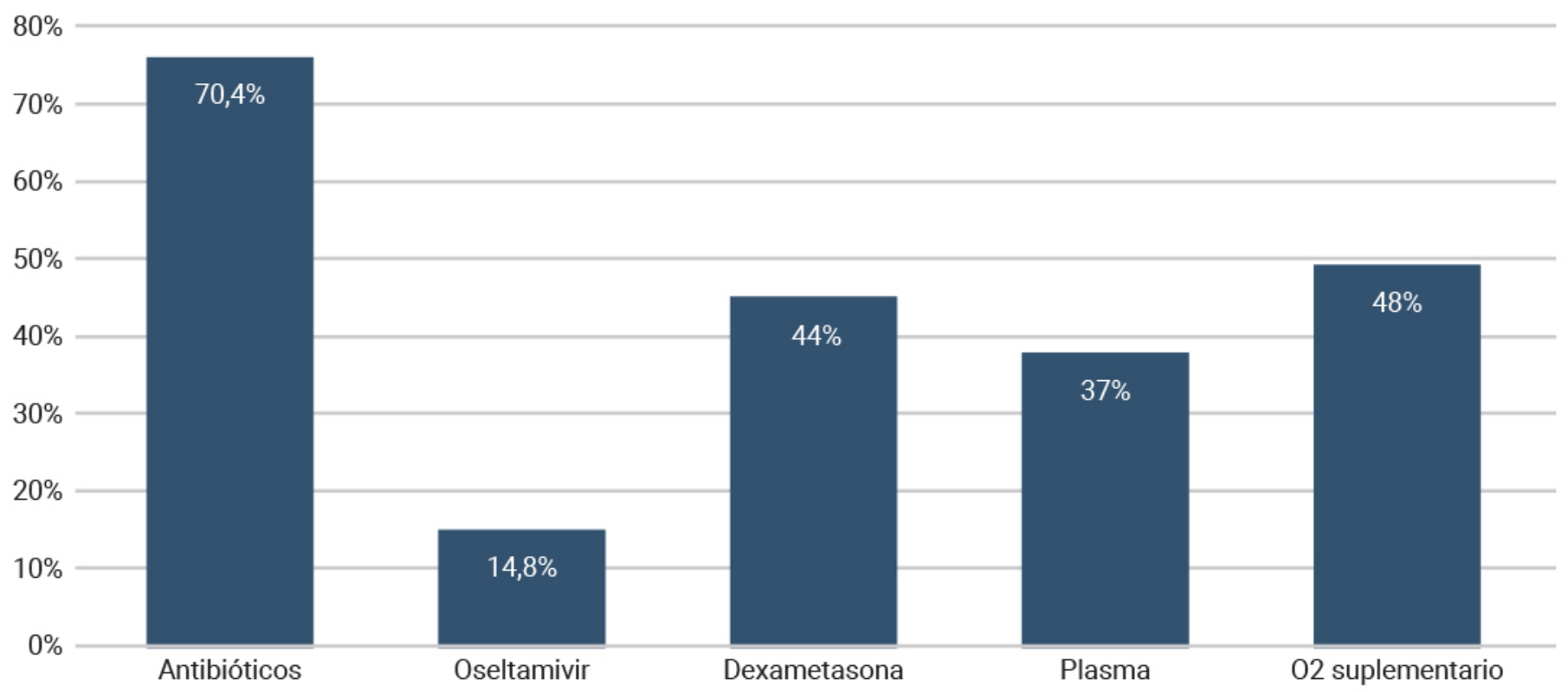

pulmón el más frecuente. Esto coincide con lo publicado en diferentes estudios multicéntricos $(8,10)$.

Una gran proporción de los pacientes adquirió la infección en la comunidad y solo unos pocos tuvieron antecedentes de contacto con enfermos de COVID-19. No obstante, un $18,5 \%$ de los casos fue de adquisición nosocomial. Pocos estudios describen la epidemiología del sitio de adquisición en pacientes con cáncer. En este sentido, y coincidente con nuestros hallazgos, en una serie de 252 pacientes con cáncer y COVID-19 Elkrief y cols. documentaron la adquisición nosocomial en el 19,1\%. En el análisis multivariado, el COVID-19 de adquisición nosocomial fue un factor de riesgo independiente de mortalidad (HR 2.28, IC 
$95 \%$ 1.28-4.05, $p=0,005$ (11). Estos datos sustentan el alto impacto epidemiológico que este virus tiene en pacientes hospitalizados con cáncer.

En cuanto a la presentación clínica, fiebre, tos y disnea fueron los síntomas más frecuentes, al igual que lo descripto en la mayoría de las publicaciones. Ningún paciente presentó los síntomas más específicos como disgeusia y anosmia, que han mostrado tener una considerable prevalencia en la población general (12). Sin embargo, nuestros hallazgos coinciden con series grandes de pacientes con cáncer en donde estos síntomas fueron muy poco frecuentes (13).

Una gran proporción de los pacientes presentaron neumonía,y a diferencia de lo publicado en pacientes sin cáncer, en donde la mayoría presenta el típico patrón de vidrio esmerilado en parches, en más de la mitad de nuestros pacientes se observó un patrón mixto o consolidativo. Nuestros hallazgos coinciden con lo publicado por Zhang y cols., en donde observaron infiltrados consolidativos en $46,3 \%$ de pacientes con cáncer y COVID-19 (14). De modo tal que en esta población debemos esperar una mayor proporción de imágenes atípicas.

A diferencia de lo que ocurre en la población general, casi la mitad de nuestros pacientes tuvieron enfermedad severa o crítica, con una proporción considerable de complicaciones e infecciones nosocomiales. Dai y cols. compararon variables de evolución en 105 pacientes con COVID-19 y cáncer, con 536 pacientes sin cáncer. Observaron que los pacientes con cáncer tuvieron mayor requerimiento de internación en terapia intensiva (OR 2.84, IC 95\% 1.49-5.08) y mayor enfermedad crítica (OR 2.79. IC95\% 1.74-4.41). Asimismo, documentaron infección hospitalaria en $19,04 \%$ contra $1,49 \%(p=<0,01)$ en individuos sin cáncer (8). De igual modo, Tian y cols. documentaron mayor proporción de enfermedad severa en 232 pacientes con COVID-19 y cáncer, comparados con 519 individuos sin cáncer ( $64 \%$ contra 32\%, OR 3.61, IC 95\% 2.59$5.04, p=<0,0001)(15)$.

La mortalidad de nuestra cohorte fue elevada, y en su mayoría asociada al COVID-19. Esto es coincidente con diferentes series en donde se informa una mortalidad de entre $13 \%$ a $28 \%(10,16)$.

Nuestro estudio tiene varias limitaciones, que mayormente están relacionadas al pequeño tamaño de la muestra. Solamente se incuyeron pacientes que requierieron internación y, de este modo, puede estar subestimada una proporción de pacientes con síntomas leves o asintomáticos que no tuvieron contacto con el sistema de salud. Si bien la mortalidad fue elevaday mayormente relacionada al COVID-19, no podemos descartar que la misma pudiera relacionarse a la edad, estadio de la enfermedad de base y a otras enfermedades preexistentes, debido a que nuestra cohorte tenía una mediana de edad elevada y una una alta proporción de pacientes en tratamiento paliativo y con comorbilidades. A pesar de estas limitaciones, nuestros datos son coincidentes con multiples series grandes y multicéntricas, que tuvieron poder estadístico para dar respuesta a estos interrogantes.

En resumen, en nuestra cohorte de pacientes con tumores sólidos, la infección por SARS-CoV-2 se manifestó mayormente como neumonía, con una alta proporción de enfermedad severa y crítica, con elevada mortalidad. Un porcentaje no menor de pacientes adquirió la infección en el hospital. Resulta fundamental implementar adecuadas estrategias de prevención tanto en la comunidad como en el hospital para disminuir el impacto de la infección por SARSCoV-2 en esta población.

\section{Reconocimientos}

Agradecemos la colaboración de los Dres. Gonzalo Recondo, Florencia Perazzo, Martín Greco y Máximo De la Vega. 


\section{Bibliografía}

1. Guan W-j, Liang W-h, Zhao Y, Liang H-r, Chen Z-s, Li Y-m, et. al. Comorbidity and its impact on 1590 patients with COVID-19 in China: a nationwide analysis. Eur Respir J 2020; 55: 2000547.

2. Richardson S, Hirsch J, Narasimhan M, Crawford JM, McGinn T, MD, Davidson KW. et.al. Presenting Characteristics, Comorbidities, and Outcomes Among 5700 Patients Hospitalized With COVID-19 in the New York City Area. JAMA 2020 May 26;323 (20):2052-2059.

3. Hussain A, Kamal Mahawar K, Xia Z, Yang W, ELHasani S. Obesity and mortality of COVID-19. Metaanalysis. Obesity Research \& Clinical Practice 2020; 14 (4): 295-300.

4. Lee L, Cazier JB, Starkey T, Briggs SE, Arnold R, Bisht V, et. al. COVID-19 prevalence and mortality in patients with cancer and the effect of primary tumour subtype and patient demographics: a prospective cohort study. Lancet Oncol 2020 Oct;21(10):1309-1316.

5. Liang W, Guan W, Chen R, Wang JL, Li C, Ai Q, et. al: Cancer patients in SARS-CoV-2 infection: A nationwide analysis in China. Lancet Oncol 2020; 21:335-337.

6. Rogado J, Obispo B, Pangua C, Serrano-Montero G, Martín Marino A, Pérez-Pérez M et. al. Covid-19 transmission, outcome and associated risk factors in cancer patients at the first month of the pandemic in a Spanish hospital in Madrid. Clin Transl Oncol 2020 Dec;22(12):2364-2368.

7. Mehta V, Goel S, Kabarriti R, Cole D, Goldfinger M, Acuna-Villaorduna A. et. al. Case Fatality Rate of Cancer Patients with COVID-19 in a New York Hospital System. Cancer Discov. 2020 Jul; 10(7): 935-941.

8. Dai M, Liu D, Liu M, Zhou F, Li G, Chen Z, et. al. Patients with cancer appear more vulnerable to SARS-COV-2: a multi-center study during the COVID-19 outbreak. Cancer Discov 2020 Jun;10(6):783-791.

9. Abdallah A, Rowland KE, Schepetiuk SK, To LB and $P$ Bardy. An outbreak of respiratory syncytial virus infection in a bone marrow transplant unit: effect on engraftment and outcome of pneumonia without specific antiviral treatment. Bone Marrow Transplantation 2003; 32: 195-203.

10. Kuderer NM, Choueiri TK, Shah DP, Shyr Y, Rubinstein SM, Rivera DR. et. al. Clinical impact of COVID-19 on patients with cancer (CCC19): a cohort study. Lancet 2020 Jun 20;395(10241):1907-1918.

11. Elkrief A, Desilets A, Papneja N, Cvetkovic L, Groleau C, Abdelali Lakehal $Y$, et. al. High mortality among hospital-acquired COVID-19 infection in patients with cancer: A multicentre observational cohort study. European Journal of Cancer 2020; 139: 181-187.

12. Lechien JR, Chiesa-Estomba CM, De Siati DRM, Horoi I, Le Bon SD, Rodriguez A. Olfactory and gustatory dysfunctions as a clinical presentation of mild-to-moderate forms of the coronavirus disease (COVID-19): a multicenter European study. Eur Arch Otorhinolaryngol. 2020 Aug;277(8):2251-2261.

13. Garassino MC, Whisenant JG, Huang LC, Trama A, Torri V, Agustoni F, et. al. COVID-19 in patients with thoracic malignancies (TERAVOLT): first results of an international, registry-based, cohort study. Lancet Oncol 2020; 21: 914-922.

14. Zhang L, Zhu F, Xie L, Wang C, Wang J, Chen R, et. al. Clinical characteristics of COVID-19-infected cancer patients: a retrospective case study in three hospitals within Wuhan, China. Ann Oncol 2020 Jul;31(7):894901.

15. Tian J, Yuan X, Xiao J, Zhong Q, Yang C, Liu B, et. al. Clinical characteristics and risk factors associated with COVID-19 disease severity in patients with cancer in Wuhan, China: a multicentre, retrospective, cohort study. Lancet Oncol 2020 Jul;21(7):893-903.

16. Lee L, Cazier JB, Angelis V, Arnold R, Bisht V, Campton NA. et. al. COVID-19 mortality in patients with cancer on chemotherapy or other anticancer treatments: a prospective cohort study. Lancet 2020 Jun 20;395(10241):1919-1926. 
Clinical and epidemiological impact of SARS-CoV-2 infection in adult patients with solid tumors

SARS-CoV-2 infection in cancer patients can be severe, and with significant mortality. We performed a prospective observational study to describe the clinical and epidemiological impact of SARS-CoV-2 infection in solid tumors patients. Twenty-seven patients with active neoplasms were included, with a median age of 72 and a median Charlson score of 6 . Infections were nosocomially acquired in $18.5 \%$ of the patients, and $74 \%$ had pulmonary infiltrates, with a high proportion of mixed and consolidative pattern in images. Almost half of the patients had a severe and critical illness. Twenty-six percent and $14.8 \%$ had complications and hospital infections, respectively. The 30 -day mortality was $25.9 \%$, mostly related to COVID-19. SARSCoV-2 infection in our population had a considerable clinical and epidemiological impact.

Keywords: infection, SARS-CoV-2, solid tumors, impact 